\title{
Adhesive wear and particle emission: Numerical approach based on asperity-free formulation of Rabinowicz criterion
}

\author{
Valentin L. POPOV ${ }^{1,2, *}$, Roman POHRT ${ }^{1, *}$ \\ ${ }^{1}$ Institute of Mechanics, Technische Universität Berlin, Berlin 10623, Germany \\ ${ }^{2}$ National Research Tomsk State University, Tomsk 634050, Russia \\ Received: 05 April 2018 / Revised: 02 July 2018 / Accepted: 31 July 2018 \\ (C) The author(s) 2018. This article is published with open access at Springerlink.com
}

\begin{abstract}
In 1953 Archard formulated his general law of wear stating that the amount of worn material is proportional to the normal force and the sliding distance, and is inversely proportional to the hardness of the material. Five years later in 1958, Rabinowicz suggested a criterion determining the minimum size of wear particles. Both concepts became very popular due to their simplicity and robustness, but did not give thorough explanation of the mechanisms involved. It wasn't until almost 60 years later in 2016 that Aghababaei, Warner and Molinari (AWM) used quasi-molecular simulations to confirm the Rabinowicz criterion. One of the central quantities remained the "asperity size". Because real surfaces have roughness on many length scales, this size is often ill-defined. The present paper is devoted to two main points: First, we generalize the Rabinowicz-AWM criterion by introducing an "asperity-free" wear criterion, applicable even to fractal roughness. Second, we combine our generalized Rabinowicz criterion with the numerical contact mechanics of rough surfaces and formulate on this basis a deterministic wear model. We identify two types of wear: one leading to the formation of a modified topography which does not wear further and one showing continuously proceeding wear. In the latter case we observe regimes of least wear, mild wear and severe wear which have a clear microscopic interpretation. The worn volume in the region of mild wear occurs typically to be a power law of the normal force with an exponent not necessarily equal to one. The method provides the worn surface topography after an initial settling phase as well as the size distribution of wear particles. We analyse different laws of interface interaction and the corresponding wear laws. A comprehensive parameter study remains a task for future research.
\end{abstract}

Keywords: adhesion; plasticity; wear; Archard's law of wear; Rabinowicz criterion; wear particle emission; surface topography; boundary element method (BEM)

\section{Introduction}

Wear is one of the most important tribological phenomena in practice, affecting the function and the life time of many mechanical systems. It is a key factor in technical safety and determines maintenance costs of many mechanical parts [1] in motion. Wear affects parts not only in machines and mechanical constructions, but also in medicine: many implants, especially artificial joints, which have to be replaced after approximately 10 years of service [2] due to wear. The critical issue with wear can be the change of shape and function of a system or the fate and behavior of the material removed in form of wear particles. Indeed, the emission of wear particles into the environment increasingly threatens human health and ecology [3]. When controlled, wear is integral to many methods of manufacturing and material processing such as grinding, polishing or sandblasting [4]. Many areas of technology and medicine are

* Corresponding author: Valentin L. POPOV, E-mail: v.popov@tu-berlin.de; Roman POHRT, E-mail: roman.pohrt@tu-berlin.de 
strongly interested in controlling wear by increasing or decreasing it depending on the particular application.

Despite its importance, wear remains one of the least scientifically understood tribological phenomena. This is in part due to the complexity of the processes influencing wear. These include contact, plasticity, crack nucleation and propagation, chemical reactions, material mixing and material transfer between contact partners and lubricants as well as the formation of surface layers. Wear is directly connected to the very broad problem of the tribological "third body". For most aspects of friction, wear is not only a consequence but also a major influencing factor [5].

For wear calculations, the most common prediction is simply that the wear volume is proportional to the normal force and sliding distance. As an empirical assumption, this law was suggested by Reye as early as 1860 [6] but it took almost a century for wear laws to be based on models of particular conditions and to be experimentally validated. In the 1950s, Kruschov conducted extensive experimental studies on abrasive wear of metals [7, 8]. Approximately at the same time, Archard carried out his classical studies of contact mechanics of rough metal surfaces in unlubricated conditions $[9,10]$ and formulated his famous wear law based on prior works of Holm and Burwell. Today this type of wear would be referred to as adhesive wear. Interestingly, both laws of Kruschov and Archard have the same mathematical form and state that the worn volume $V$ is proportional to the normal force $F_{\mathrm{N}}$ and the sliding distance $s$ and inversely proportional to the hardness $\sigma_{0}$ of the softer material:

$$
V=k \frac{F_{\mathrm{N}} s}{\sigma_{0}}
$$

The constant $k$ is the so called wear coefficient, whether adhesive or abrasive.

In the case of abrasive wear, the wear coefficient has a relatively clear physical interpretation based on the idea of micro-cutting of the softer material by the asperities of the harder counterpart [11]. The coefficient of abrasive wear is thus relatively well defined and ranges from approximately $10^{-3}$ to $10^{-2}$. It is least when "three-body" wear occurs, e.g., two metallic parts with loose abrasive particles in between them, and is most severe for "two-body" abrasive wear, like a metal piece in contact with sandpaper [11].

In the case of adhesive wear, no clear physical interpretation of the wear coefficient exists. Archard did only suggest a first idea of the probability that two impacting asperities will form a wear particle. In the following 50 years, there was only little progress in formulating physical, model-based criteria for the adhesive wear coefficient. Empirically measured values of the adhesive wear coefficient are known to differ by 5 decimal orders of magnitude and also to depend on the type of materials [11]. This fact makes Eq. (1) a very poor predictor for the wear behavior of a general or unknown tribologial system. While the dependencies on the normal force and the sliding distance may be approximately valid for any given system in a limited range of loads, the inverse proportionality of wear volume to hardness loses direct sense. There is a widespread opinion that the higher the hardness, the lower the wear, because the hardness is in the denominator of Archard's equation. However, Kragelsky [12] formulated the exact opposite principle for minimizing wear-the principle of a positive hardness gradient. According to it, the hardness at surface layers must be less than inside the bulk body in order to avoid catastrophic wear. In Ref. [13], more complicated dependencies of wear rate on hardness are discussed, including inverse dependencies. A very good review of different modes of wear as well as transitions between mild and catastrophic wear is given in Ref. [14] with the conclusion that "no single predictive equation or group of limited equations could be found for general and practical use". Regrettably, this conclusion, made in 1995, remained mostly valid until today.

In the last few years some ideas emerged which allow a new understanding of the physics of wear. As happens so often in the history of science, recent developments just shed new light on old principles. Aghababaei, Warner and Molinari (AWM) [15] used an artificial model potential to enable simulation in relevant length scale in the frame of quasi-molecular dynamics simulations. Dimaki et al. [16] implemented a mesh-dependent fracture criterion to achieve the same in the discrete element method.

The findings of AWM confirmed an old idea that 
was put forward by Rabinowicz in 1958 [17]. In the criterion of Rabinowicz and the AWM simulations, the interplay of plasticity and adhesion leads to the emergence of a characteristic length controlling wear: If a micro contact is smaller than the characteristic length, then it will be plastically deformed; if it is larger than the characteristic length, a wear particle will emerge. The existence of these two scenarios has also been observed in recent molecular dynamics simulations [18]. Combined with advanced numerical simulation methods of contact between rough surfaces [19], this new understanding advances the old idea of Rabinowicz to a new paradigm [19,20] in the science of wear. Frérot, Aghababaei and Molinari [21] re-interpreted Archard's probability of a given wear particle to actually emerge as the probability to encounter an asperity contact capable of forming a wear particle, according to the deterministic RabinowiczAWM criterion [20]. For the first time this concept allowed a microscopic, model-based understanding of the adhesive wear coefficient.

While this concept has enormous attractiveness, it also has an essential shortcoming: just as the original Rabinowicz criterion, it is based on the analysis of a single "asperity". However, it is widely recognized that the notion of asperity is poorly defined for real surfaces having roughness on many length scales. Molecular dynamic simulations of only two neighboring surface peaks show that they can behave as one single asperity if the distance between them is small enough [21, 22]. The authors of Refs. [23, 24] attempted, based on the idea of Rabinowicz, to formulate an asperity-free criterion of forming wear debris. The present paper can be considered a practical numerical implementation of the ideas formulated in Refs. $[20,23,24]$. Based on the asperity-free generalized Rabinowicz criterion, we develop a model describing the formation of wear particles and the evolution of the surface topography.

The paper is organized as follows. In Section 2, we shortly recapitulate the original arguments of Rabinowicz as well as the concept of the asperity free wear criterion formulated in Refs. [23, 24]. In Section 3, we use the asperity-free concept for designing a model of wear processes. Section 4 gives the results of simulations using the formulated wear model. In
Section 5, we discuss the transitions between the stages least wear, mild wear and severe wear which have already been discussed by Rabinowicz and are also found in our simulations. Finally, in Section 6, we present the main conclusions.

\section{Generalized Rabinowicz criterion for formation of wear debris}

We start with the reproduction of the original Rabinowicz criterion [11, 17, 25]. Consider two micro heterogeneities colliding and forming a welded bridge, as suggested in the well-known generic picture of dry friction suggested by Bowden and Tabor [26] (see Fig. 1).

The maximum stress that can be achieved in the welded contact is of the order of the material hardness $\sigma_{0}$. The stored elastic energy $U_{\mathrm{el}}$ can thus be estimated as the product of characteristic energy density $\sigma_{0}^{2} /(2 G)$ and the characteristic stressed volume $D^{3}$ :

$$
U_{\mathrm{el}} \approx \frac{\sigma_{0}^{2}}{2 G} D^{3}
$$

where $G$ is the shear modulus and $D$ the characteristic size (diameter) of the joint. If a wear particle was formed, this energy would relax. The process of detaching a wear particle can only occur if the stored elastic energy exceeds the energy needed to create new free surfaces

$$
U_{\mathrm{adh}} \approx \Delta w \cdot D^{2}
$$

Here $\Delta w$ is the work of adhesion per unit area. It follows that only particles larger than some critical size $D_{\text {crit }}$ can be detached:

$$
D>D_{\text {crit }}=\frac{2 G \cdot \Delta w}{\sigma_{0}^{2}}
$$

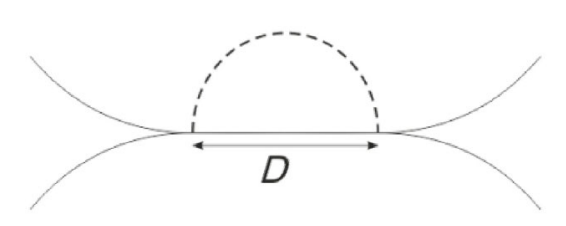

Fig. 1 Welded joint of size $D$ created due to impact and consecutive shear of two asperities. 
Note that this equation predicts only the existence of a lower bound of the size of wear particles. Possible mechanisms suppressing the appearance of too large particles will be discussed later in this paper (see Sections 3.3 and 5.).

According to Rabinowicz, the key quantity governing the formation of wear particles is $D$, the "asperity size", which is poorly defined for real surfaces having fractal character [27]. Looking at a contact configuration of bodies with fractal rough surfaces as shown in Fig. 2, we see loosely connected clusters of contact areas instead of separated asperities. A thoughtful look at the logic behind the Rabinowicz criterion reveals that the notion of a single asperity is in fact not necessary for the application of this logic. Below we describe the basic idea (as first suggested in Refs. $[23,24])$ of how an energy based wear criterion can be formulated without it.

In Fig. 2, the contact of an elastic half-space and a rough surface is shown. The topography was generated as described in Ref. [28] with roughness having the Hurst exponent 0.7. Using the BEM implementations as described in Ref. [19] or Ref. [29], the stress distribution at the interface can be calculated according to a particular assumption for the interaction in the contact surface, e.g., a constant coefficient of friction, some given tangential flow stress or adhesive interaction. In Fig. 2(b), the distribution of normal stress is shown but similar distributions can be easily produced for all stress components.

The central idea of the generalized Rabinowicz criterion is the following: Consider a circular region with arbitrary diameter $D$ centered at an arbitrary point of the contact interface. In Fig. 2(b), several examples

(a)

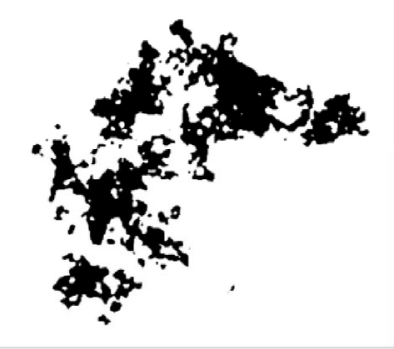

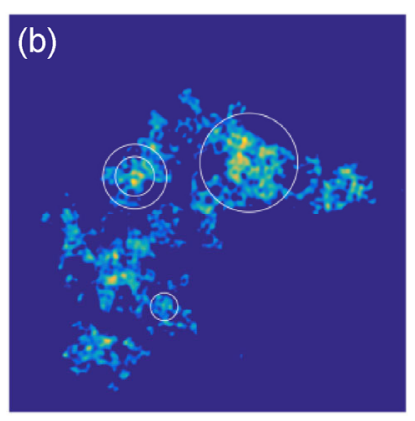

Fig. 2 Numerical simulation of the normal contact between a rough surface and an elastic half-space: (a) contact area at a given indentation depth; (b) pressure distribution in the contact. of such regions with different positions and different diameters are shown with white circles. If a wear particle of diameter $D$ is formed at that particular position, then the surface stresses in the chosen region will vanish. This leads to the release of the elastic energy $U_{\text {el }}$ which can be estimated with the same BEM program (description of procedure see next section). Following the logic of Rabinowicz, the wear particle can only be formed if

$$
U_{\mathrm{el}}>\frac{\pi}{4} D^{2} \Delta w
$$

Thus, probing each surface point with circles of varying diameter, we can always assess whether the formation of a wear particle at the given position and with the given size is possible. Note that this criterion is completely independent on the notion of "asperity". It can be applied to an arbitrary contact configuration, stress distribution and even to positions which are not part of the actual contact zone. Equation (5) identifies parts of material that can detach due to the elastic energy stored in the corresponding zone. Just like the original Rabinowicz criterion, Eq. (5) does not state whether the process of detachment will really take place. However, molecular simulations of AWM [15] strongly suggest that it is reasonable to equate the possibility and the actual occurrence of wear debris formation: In the following, we will assume that particles which can be produced energetically will indeed realize this possibility. In this way, we obtain a constructive rule to the formation of wear particles and thereby to the process of surface evolution.

\section{Minimalistic model of adhesive wear}

In this section we describe a simplified, minimalistic model of adhesive wear. Let us start with the contact of a rigid plane surface and an elastic body having a periodic, fractal rough surface characterized by the Hurst Exponent $H$, lower and upper cut-off wavelengths $\lambda_{\text {long }}, \lambda_{\text {short }}$ and the rms roughness $\theta$. The bodies are first brought into normal contact with average pressure $\tilde{p}$ and then forced into a continuous tangential movement relative to each other. In the present study, we restrict ourselves to purely elastic 
normal contact solution-up to the moment of formation of a wear particle. However, the rule for tangential stresses in the interface can be different, for instance it can be based on Coulomb friction or on the assumption of a limiting flow stresses. As was illustrated in Ref. [30], the interface "flow law" can mimic to some extent the material behavior, e.g., plasticity.

The wear model is based on the analysis of the stress distribution in the contact. Only the tangential stress components will be taken into account in this analysis, because pure normal contact does not allow opening cracks-independently of the stress intensity. It is the tangential stress on the surface which is capable of creating conditions for crack nucleation. In the simulations of AWM [15], tangential stress applied to the surface leads to the appearance of tensile stress in a plane inclined approximately $45^{\circ}$ to the surface at the trailing end of the contact region; this is what enables crack opening. Of course, the appearance of tensile stress is only one necessary condition for the formation of a wear particle. It is also indispensable that the crack should be able to propagate. This is only the case if enough elastic energy is stored in the material for producing fresh surfaces as described by the Rabinowicz criterion. In the framework of the boundary element method, we do not have the possibility to investigate the process of crack propagation. Following the idea of Rabinowicz-AWM [15], we just assume that if the two above conditions (appearance of tensile stresses and energetic possibility of crack propagation) are fulfilled, then the wear particle will form.

Of course the problem of wear cannot be reduced solely to the formation of wear particles. True wear occurs only when particles have been transported out of the friction zone $[31,32]$. During this process they can be integrated again into the surfaces or take part in the process of mass mixing and the formation of surface layers [33]. All these processes are not considered in the present study due to two reasons. First, it is our intention to concentrate in detail on the initial and fundamental sub-process of wear, knowing it is only one part of the series leading to the phenomenon "wear". Second, there is currently no simple numerical tool available to convincingly simulate the complete chain of wear sub-events. In this study we thus assume that any formed wear particle will instantly disappear. We are fully aware that in many cases this assumption will be too strong to achieve a correct quantitative prediction of wear but we hope to give a correct qualitative understanding of the main governing parameters of the wear process.

Based on the above explanations, we suggest the following wear model consisting of two main steps.

\subsection{Step 1: Determining the location of wear particle initiation and corresponding size}

In this first step, the tangential stress distribution is scanned using the criterion (5). Let us discuss this important step in more detail. By dividing (5) by the area $A=\pi / 4 D^{2}$ of a circle with diameter $D$, we can rewrite this criterion in the form

$$
E=\frac{U_{\mathrm{el}}}{A}>\Delta w
$$

stating that the condition for appearing a wear particle at some position is that the elastic energy density, $E=U_{\mathrm{el}} / A$ should exceed the work of adhesion per unit area. We thus start by constructing maps showing the energy density at any position in the contact area. Note that the energy density map should be calculated separately for any diameter $D$. For the underlying tangential stress, some particular flow law in the interface must be assumed. The following simulations in this section are carried out under the assumption that tangential stress in the contact plane is determined by the Coulomb friction law with a coefficient of friction $\mu$ and continuous sliding in all contact points. This translates into the tangential stress distribution $\tau$ following the distribution of pressure $p$ :

$$
\tau(\vec{x})=\mu p(\vec{x})
$$

where $\vec{x}$ is a position in the contact plane.

In the framework of the BEM simulation, each calculation step gives the complete distributions for stresses $\tau$ and displacements $u$. The energy which is released by relaxing the stresses inside a circle with diameter $D$ can be readily calculated as

$$
U_{\mathrm{el}}(\vec{x})=\frac{1}{2} \int_{A_{\text {Circle, },}}\left[u(\vec{x}+\vec{r})-u^{(0)}(\vec{x}+\vec{r})\right] \tau(\vec{x}+\vec{r}) \mathrm{d}^{2} \vec{r}
$$


where superscript.${ }^{(0)}$ refers to the stress free state. The integration is carried out over the area of the chosen circle. Scanning point by point the complete computational area, calculating the integral (8) and dividing it by the area $A_{D}$, we can produce energy density maps for each position of the circle center as shown in Fig. 3.

The energy density map depends of course on the contact configuration (and thus on the normal load) and on the diameter $D$ of the "probing circle". Let us first consider the dependency on the diameter. If we take some point in the region of real contact, then increasing $D$ may first lead to increasing the energy density, since the elastic energy stored in a region with homogenous stress is roughly proportional to $D^{3}$. With further increase of the diameter $D$, the circumference of the circle may leave the region of contact. The stored energy will not increase further, but the denominator $A$ in Eq. (6) still increases, so that the energy density $E$ starts to decrease. We can conclude that when the contact configuration is composed of distinct islands which are not or poorly connected, then the energy density is not a monotonic function of diameter $D$. This is valid both for each particular point and for the maximum value of the energy density, which is of main interest as it determines the first point of detachment of a wear particle.

To accelerate the construction of the energy density map, we used also an approximate measure. We first determine the average stress in the probe circle $\tilde{\sigma}_{D}$ which to find is a very simple analytical procedure. The energy density $E_{\mathrm{D}}$ stored due to constant tangential stress in a circle with diameter $D$ is given by

$$
E_{\mathrm{D}}=\frac{4 D}{3 \pi G^{*}} \tilde{\sigma}_{D}^{2}
$$

where $G^{*}=4 G /(2-v)$ is the effective shear modulus.

The results of the evaluation are shown in Fig. 4 in which the normalized energy density $E_{\mathrm{D}} / \Delta w$ is represented as function of $D$. The shaded area shows the complete interval of energy densities which have been observed in the contact area. Of the main importance is of course the upper boundary of the shaded area showing the maximum energy density for a given $D$, because it identifies the position where the condition for forming wear particles is first satisfied. We also show the values of the exact evaluation using Eq. (8) with the black line. When the maximum of the normalized energy density is smaller than 1 , formation of wear particle is energetically impossible at any position.

\subsection{Step 2: Formation of wear particles}

With increasing normal force, the maximum energy density will also increase and the dependency of the normalized energy density on $D$ will touch at some moment the horizontal critical line $E / \Delta w=1$. The point and the size at which this happens determine unambiguously the position and the size of the wear
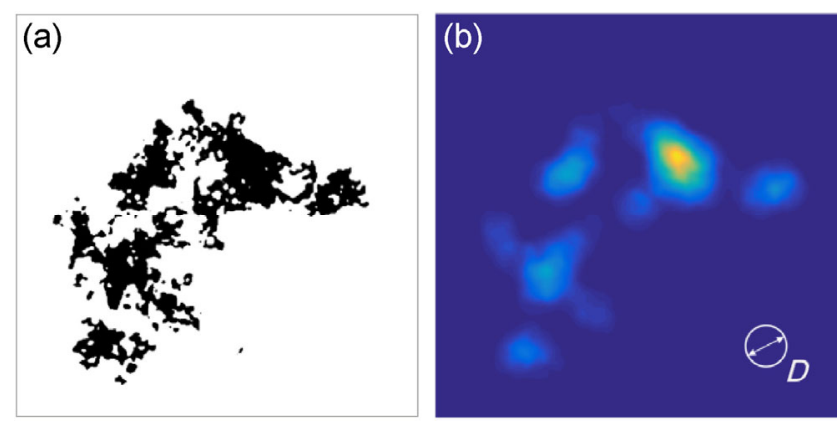

Fig. 3 (a) Contact configuration and (b) the corresponding energy density map for a given diameter $D$.

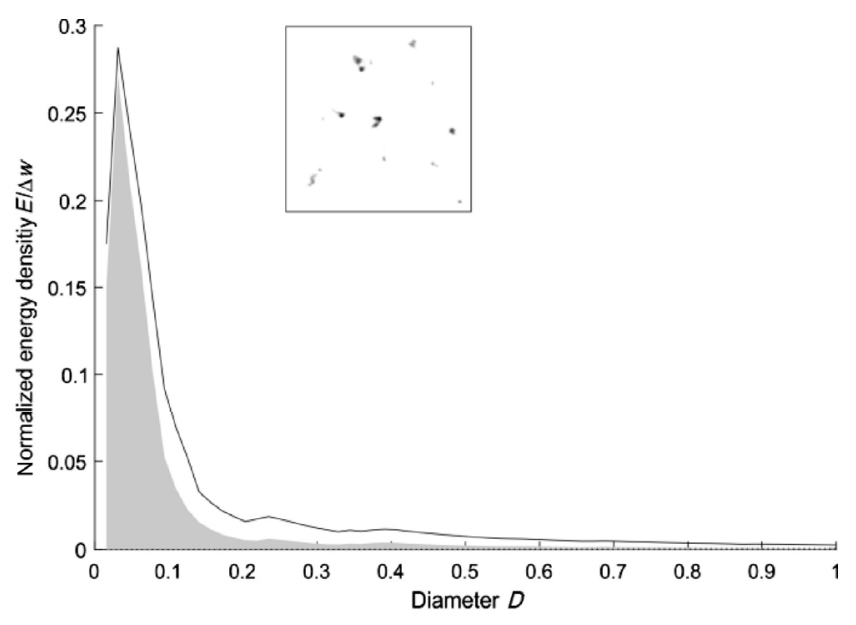

Fig. 4 Dependency of the energy density at the given contact configuration as a function of the diameter $D$ of probing circles. The grey zone is limited by the minimum and maximum values that occur. The contact configuration which has been analyzed is shown in the insert. It was generated using the following parameters: $G^{*}=1, v=0, H=1, L=1, \lambda_{\text {long }}=1 / 3$, $\lambda_{\text {short }}=1 / 32, \tilde{p}=1 / 10, \Delta w=1, \mu=0.3, \theta=0.4$. 
particle which will be formed. The corresponding wear particle inside the circle with diameter $D$ is then extracted, such that the surface topography is altered from $z_{\vec{x}}$ to $z_{\text {new }, \vec{x}}$ according to

$$
\begin{aligned}
& z_{\text {new }, \vec{x}}(\vec{r})=z_{\vec{x}}(\vec{r}) \\
& \quad+\sqrt{1-4|\vec{r}|^{2} D^{-2}} \cdot\left[\tilde{z}_{\vec{x}, D}-z_{\vec{x}}(\vec{r})-d_{D}\right] ; \\
& d_{D}=5 \theta\left(0.5 D / \lambda_{\text {long }}\right)^{H}
\end{aligned}
$$

Where $\tilde{z}_{\vec{x}, D}$ is the mean height inside the circle region. We chose this particular procedure to ensure that the new surface geometry remains continuous. Figure 5 shows an example of a surface geometry before and after the particle removal. This act closes one cycle of the wear calculation. With the new topography, the stress calculation and energy density evaluation is carried out again and the position and size of the next wear particle is identified, which is detached and extracted again until $E<\Delta w$ in every point.

\subsection{The problem of "very large" wear particles and its handling}

The described procedure of finding the critical region where a wear particle will be detached following Eq. (6) has a large-particle-paradox. At small normal forces, the contact consists of distinct contact spots as shown in Fig. 4 and the maximum energy density has a pronounced maximum when plotted over $D$. At higher forces however, the picture changes and resembles Fig. 6.

One can see that when the fraction of area being in contact becomes larger, the energy density in regions with larger $D$ grows. This is an immanent property of any contact of periodic and thus macroscopically flat surfaces. Whatever the particular stress distribution, for large enough probe circles, the surface deformation
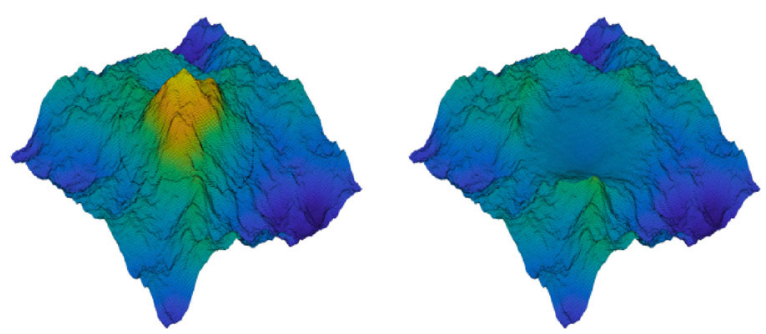

Fig. 5 Topography of a rough surface before and after detachment and disappearance of a wear particle.

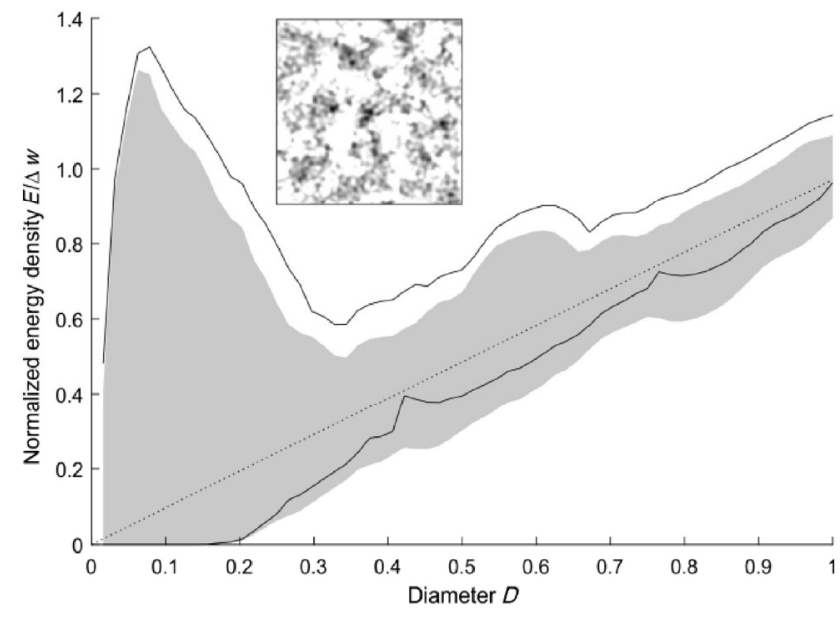

Fig. 6 Illustration of the energy density curve at high forces. Large diameters of probe the circle necessarily lead to large values of $E$. The mean pressure was chosen as $\tilde{p}=5$, other parameters as in Fig. 4.

and resulting elastic energy inside them resemble the case of homogenous constant stress. The elastic energy scales as $D^{3}$ and the corresponding energy density scales as $D$ : it increases linearly with the diameter. Inserting the system's global average stress $\tilde{\sigma}_{\text {sys }}$ into Eq. (9) we can always find a minimum diameter, where the energy density equals or exceeds the work of adhesion, thus satisfying the detachment criterion. Also one can always find a circle inside the apparent contact area that satisfies $\tilde{\sigma}_{D} \geq \tilde{\sigma}_{\text {sys }}$. Therefore a catastrophic diameter $D_{\text {cat }}$ dependent on the eternal load must exist, which is guaranteed to include enough energy to generate a wear particle.

$$
D_{\text {cat }}=\frac{3 \pi}{4} \frac{G^{*} \Delta w}{\tilde{\sigma}_{\text {sys }}^{2}}
$$

This "problem of large particles" is not new; it existed already in the original Rabinowicz criterion, which predicts only the existence of a lower bound of the size of wear particles. A possible mechanism suppressing the appearance of arbitrary large particles was suggested in Ref. [30] and is based on the Rabinowicz criterion itself. If some particle has a size much larger than prescribed by the Rabinowicz criterion (4), then it is energetically favorable for it to disintegrate into smaller ones. This means that particles with the size substantially larger than the Rabinowicz-AWM critical length can never practically 
appear. In the present study we enforced this property by formally bounding the particle size by a fixed maximum value.

\section{Wear process and development of worn surface topography}

Depending on the initial topography, the wear properties of contact partners and the character of interaction in the interface, two basic scenarios of wear are possible. In the first scenario, the wear process alters the surface topography in such way that after an initial phase, no more wear occurs. Instead, the new topography makes the applied load distributed in such a way, that our local wear criterion cannot be satisfied anywhere on the surface. Because of the foregoing wear process leading to new steady-state of no wear, we will refer to this scenario as the "settling" type of wear. The general principle of a surface geometry changing towards a state of no-wear is known from fretting wear. Here small oscillatory tangential displacements wear off material where it is most harshly stressed [34, 35].

On the other hand, one can have continuously proceeding wear. We start with an example of the settling type of wear.

\subsection{Wear of "settling" type}

We consider the contact between a wear-resistant smooth plane and a rough counterpart where the wear takes place. In the contact interface, we assume Coulomb friction and complete sliding, so that the tangential stress at the surface is proportional to the local normal stress, see Eq. (7). The elastic energy stored in a circle of diameter $D$ with average normal pressure $\tilde{p}_{D}$ can be estimated with the help of Eq. (9)

$$
E_{\mathrm{D}} \approx \frac{4 D}{3 \pi G^{*}}\left(\mu \tilde{p}_{D}\right)^{2}
$$

As one of the counterparts does not change its configuration, a situation is possible that after a number of particle detachment events, the stress will be re-distributed in such a way that no further particles can form. In this case, wear occurs solely during a finite time of "settling". Afterwards, the surface topography remains stable. Figure 7 shows a sample surface before and after being subjected to such wear process. Because the opposing flat surface does not wear out, no directional dependence is visible in the wear marks of the worn rough surface in Fig. 7(b).

In every step, we record the sizes of the particles for analysis. With assumed Coulomb law of friction in the interface, the stresses are not bounded by plastic deformation. Thus, arbitrarily small particles can be produced. Simulations show that most of the particles are indeed of the minimal size, given by the grid resolution. Looking at the distribution of the worn volume $V$, estimated as a spherical particles

$$
V=\sum_{D} n_{D} \frac{4}{3} \pi D^{3}
$$

we find a more equal dependency, see Fig. 8. Here $n_{D}$ is the number of particles with size $D$.
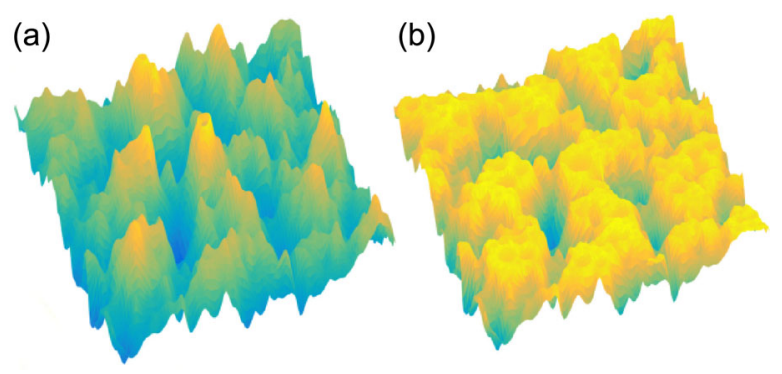

Fig. 7 Illustration of a rough surface (a) and its worn state (b) according to the rules described in Sec. 4.1. It was generated using the following parameters: $G^{*}=1, v=0, H=1, L=1$, $\lambda_{\text {long }}=1 / 3, \quad \lambda_{\text {short }}=1 / 32, \quad \theta=0.4, \tilde{p}=1 / 2, \Delta w=1 / 100$, $\mu=0.3$.

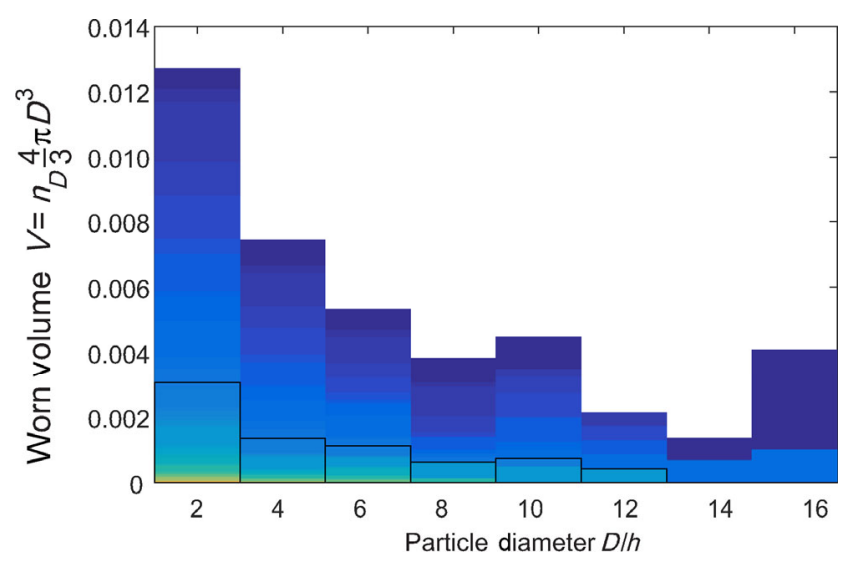

Fig. 8 Histogram of the wear volume of 6986 particles as a function of the particle size (given in terms of the computational grid spacing $\Delta$ ). No particle larger than $D=16 \Delta$ occurred. Different colors mark different force levels up to $\tilde{p}=1 / 2$, see the black boxed series for instance, other parameters as in Fig. 7. 
In the final state, both the topography of the worn surface and the overall wear volume depend on the normal loading. In this study we will confine ourselves to the analysis of the total worn volume as a function of the increasing applied load. This is given in Fig. 9. The worn volume can be approximated by a powerlaw dependency of the average pressure with the exponent 1.4:

$$
V \propto \tilde{p}^{1.4},
$$

See the dotted line in Fig. 9.

\subsection{Continuous wear in contact of two rough surfaces}

As a second example, let us consider the same problem as in the preceding section with the only difference that now the rough body is rigid and the smooth counterpart wears out. As before, we assume the tangential stresses to be proportional to the normal pressure. In numeric experiments a rough rigid surface was moved tangentially over an initially smooth surface.

We investigated the worn volume for the same rough topography for a series of increasing normal pressures. Figure 10 shows resulting topographies of this process for two different average pressures. For each time step corresponding to a certain distance covered, we recorded the worn volume of all particles that were generated. Figure 11 shows a typical evolution of the worn volume. It is practically linear with the

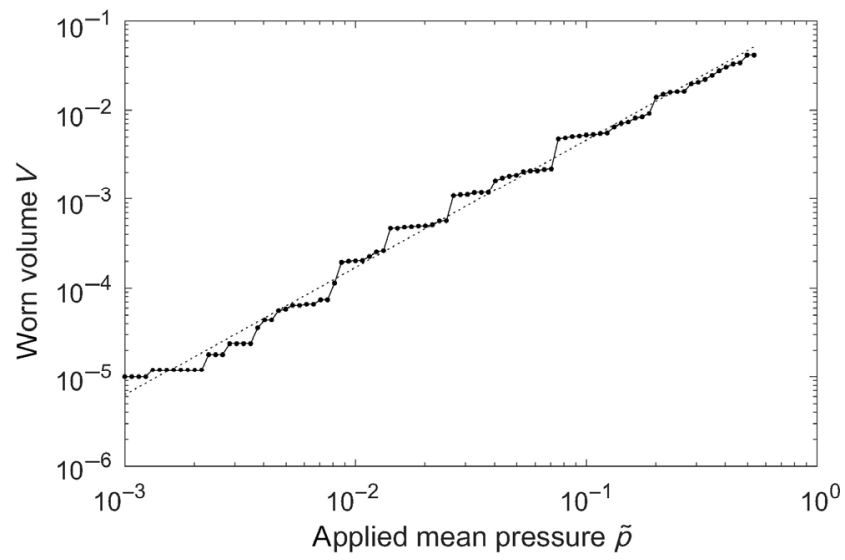

Fig. 9 Dependency of the settled worn volume as a function of the applied external load. In this case, the wear volume does not depend on the distance covered but occurs instantaneously for any given load, parameters as in Fig. 7.
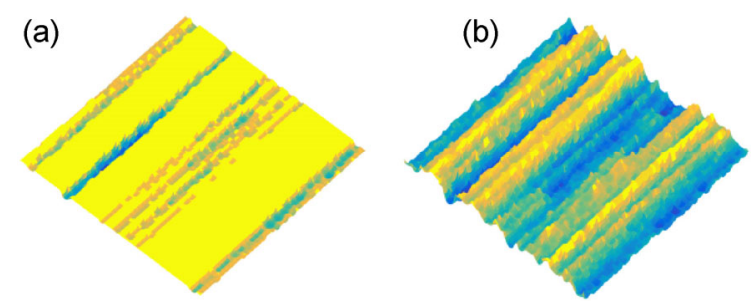

Fig. 10 Two initially smooth surfaces after being subjected to wear as described in Sec. 4.2 with a rough counterpart similar to Fig. 7(a). (a) At light load $\tilde{p}=0.01$, many contacts slide but do not generate particles and the surface remains undamaged at these spots. (b) With more severe loading at $\tilde{p}=0.1$, a larger part of the surface is worn. Other parameters as in Fig. 4.

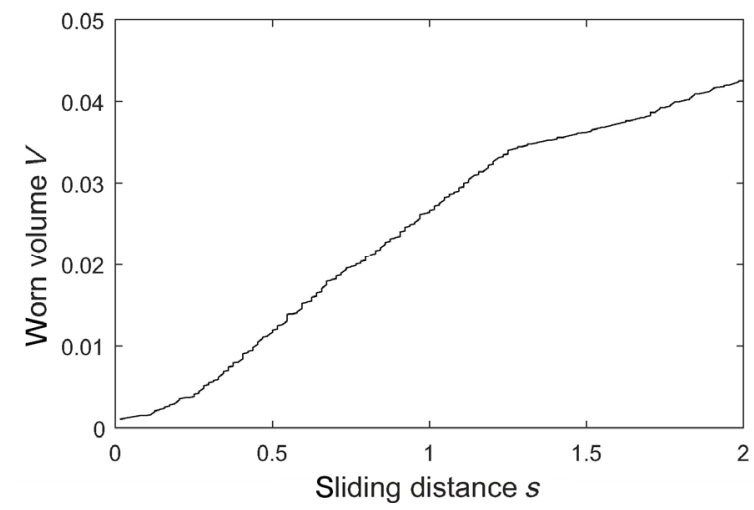

Fig. 11 Dependency of the worn volume as a function of the sliding distance covered. Mean pressure was $\tilde{p}=1$ (circled value in Fig. 12), other parameters as in Fig. 4.

factor depending on the exact contact configuration in the corresponding instant. We expect the behavior to be more homogenous with smaller $\lambda_{\text {long }}$, when a greater number of isolated peaks determine the surface topography. Averaging over the complete distance covered $(2 L)$ one can define a specific worn volume per unit length. Figure 12 displays the corresponding dependency on the applied mean pressure and a power-law fit. We found the wear volume worn by distance to scale as

$$
V \propto \tilde{p}^{1.77}
$$

which strongly deviates from Archard's and Kruschev's law.

Note that the worn surface (Fig. 6(b)) has pronounced grooves.

This appearance is usually considered a sign of abrasive wear. However, in the present case we consider pure adhesive wear: there are no processes like micro-cutting or ploughing. 


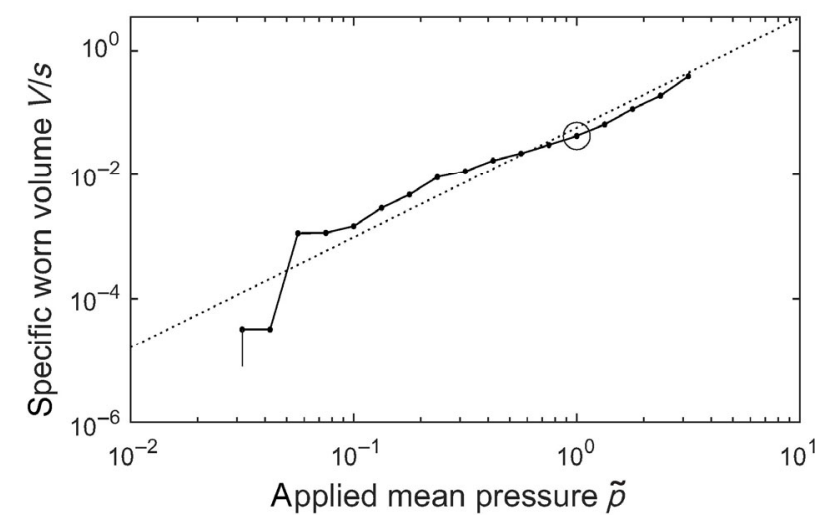

Fig. 12 Dependency of the wear volume per distance as a function of the applied external load. Parameters as in Fig. 4.

\subsection{Wear of a rigid rough surface and a rough elastic surface with constant tangential flow stress in the contact region}

In this section, we assume that tangential stress in the contact is constant and equal to $\sigma_{0}$. This case is the most important so far as it mimics most correctly the mechanism of wear of ductile materials like metals. The assumption of a constant flow stress mimics the behavior of elastic-ideally plastic materials [30]. Due to the final flow stress, tangential stresses are now bounded by $\sigma_{0}$, in much the same way as assumed in the criterion of Rabinowicz. In this case we again have continuous wear. The wear volume, normalized by distance covered is shown in Fig. 13 as a function of the applied load.

After achieving the conditions for wear for the first time, the wear volume increases continually with load. This is the classical region of adhesive mild wear. In this region, the worn volume follows the power-law dependency

$$
V \propto \tilde{p}^{1.36}
$$

Note that because the wear volume is not proportional to the normal force, Archard's law is not directly confirmed. However, the exponent 1.36 is close to one and in some finite force intervals the dependency can be considered as approximately linear. Indeed, the non-linear dependencies of worn volume on the normal force in the case of large force interval are well known and have been reported as early as the 1970s [36].

\section{Transitions between least wear, mild wear and severe wear}

Let us discuss the transitions between the three stages least wear, mild wear and severe wear, as introduced by Rabinowicz [37]. The condition of least wear has a simple physical meaning which basically was already described by Rabinowicz [11]. In the case of a finite flow stress as described in Sec. 4.3, the Rabinowicz characteristic length is directly applicable. If the bodies are pressed against each other at light load, the contacts will typically have under-critical size compared to the Rabinowicz length and will not wear. In our framework, this translates to the normalized energy density being below 1 at any point for any $D$. In this region, it is very unlikely for a contact of sufficient size to occur and in the numerical model, it might not happen at all. Consequently, only least wear can occur. In Fig. 13, this region of loads is shown on the left side.

At higher loads, the contact configuration becomes dense, and the transition to mild wear (central field in Fig. 13) begins. As we enter the true region of contact of rough surfaces, the statistical properties of the contact start to dominate. For this region, a power-law dependency of the worn volume on the normal load is characteristic.

Finally, at very high normal loads, larger particles form, as elaborated in Sec. 3.3. We interpret this as the

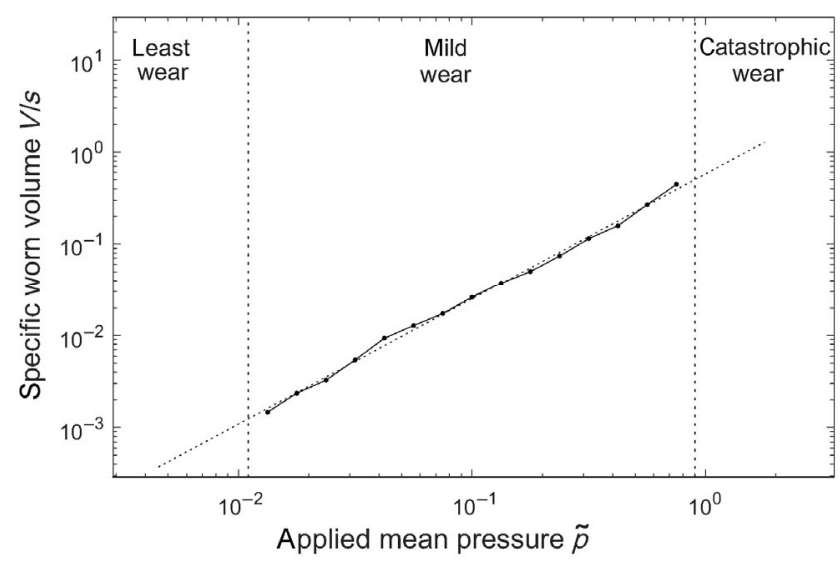

Fig. 13 Dependency of the wear volume per distance as a function of the applied external load for a constant flow stress $\sigma_{0}=10$. Other Parameters as in Fig. 4. For very low loads, no wear occurs in the model (least wear mode). At very high loads, very large particles emerge which the model cannot predict. 
beginning of "catastrophic wear" (right field in Fig. 13). Because larger particles are to be expected, the question is rather why they do not occur than why they occur. In our simulation we assumed that once a particle of the size comparable to the computation area is possible, wear is catastrophic.

However, this maximum size is set somewhat arbitrarily. The physical understanding of the correct setting of this quantity is still an important future task and will surely be the key to physical understanding of catastrophic wear.

One possible approach follows directly from the concept presented in this paper: the energy density as function of particle diameter. For any wear particle to be formed, it is necessary that the stress distribution generates some tensile stresses and the elastic energy stored must be enough for separating the surfaces. However, there is an additional restriction for the possibility of wear particle formation. In a multicontact-configuration, a particular wear particle can only be detached if it "fits inside the gap". No particle can emerge with diameter greater than the current gap between the surfaces, because it would have to increase the global clearing. It is assumed that no single particle can do that. Considering this, the gap itself is comparable to the existing wear particles. In the case of the energy density of the form shown in Fig. 6, our energetic criterion allows the formation of particles corresponding to the first maximum and in principle also those corresponding to the right end of the linear asymptote. In reality, only the particle corresponding to the first maximum will be formed as those corresponding to the linear asymptote are too large and do not fit in the gap. The situation changes at larger normal loads as exemplarily shown in Fig. 14. Now the first maximum has shifted towards the region of larger diameters and finally merges with the linear asymptote which has also risen. This qualitatively changes the situation. Now there is no distinct small-particle-size capable to controlling the gap and thus preventing larger particles from being formed. Starting with smaller particles, wear can now continuously migrate to the formation of larger and larger particles while continuously increasing the gap size at the same time. In the framework of our model, this process is unbounded and indeed catastrophic.

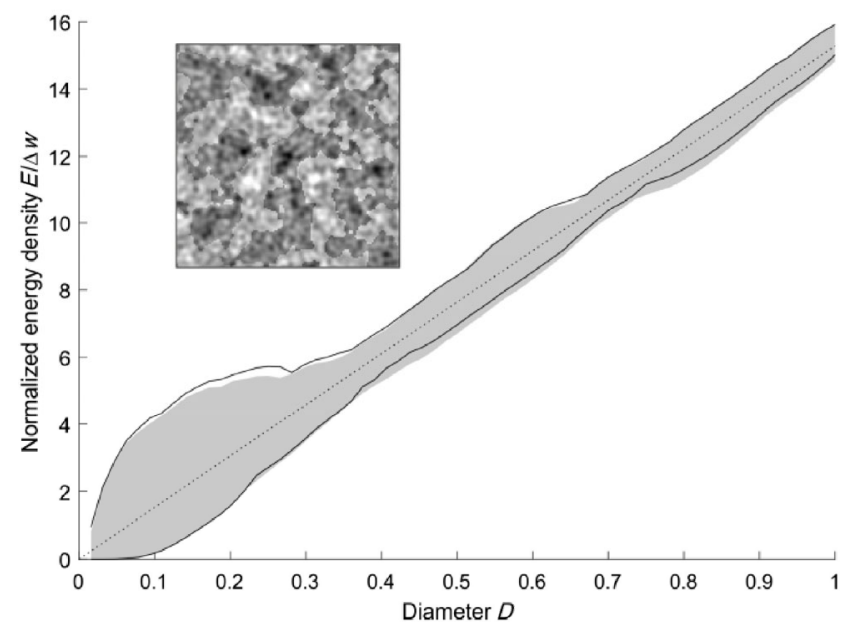

Fig. 14 Transition between bi-modal and uni-modal energy density may be the mechanism of transition to catastrophic wear. The mean pressure was chosen as $\tilde{p}=20$, other parameters as in Fig. 4.

We consider the validation of the above principle an important step for future research. In order for it to be checked and validated, a more extended model, describing both the stages of particle formation and their further transport is required.

\section{Discussion and conclusion}

This paper is devoted to the generalized applications of principles suggested by Archard and Rabinowicz more than 50 years ago. In this sense, the title of the paper could be: "Archard and Rabinowicz revisited". Archard emphasized the importance of roughness but at his time he had no possibility to carry out detailed contact mechanics simulations. At present, we can realize his ideas in numerical experiments for particular surface geometries. Similarly, Rabinowicz suggested a very general but also very vague idea about his criterion for the detachment of wear particles. His criterion became a veritable theory only after the recent breakthrough research by AWM. But even in their concept, the central notion of "asperity" remained unclarified. In the present paper, we use the recently suggested concept of an asperity-free wear criterion. As a matter of fact, this criterion generalizes the idea of Rabinowicz by noticing that the notion of asperity is in fact dispensable in the framework of its logic! With the completion of this missing link, all parts of the Archard and Rabinowicz concepts can be 
implemented using numerical contact mechanics.

In the present paper we numerically implemented the above ideas, tested the new numerical concept for wear simulation and provided illustrations of how the concept works in various situations. Already these illustrations give an idea of what the main governing parameters of wear may be, but extensive parameter studies and a deeper understanding of the governing parameters will be necessary in the near future, as it was not the intention of the present paper.

Our numerical simulations based on the asperity-free generalized Rabinowicz criterion show that the wear regimes can depend on properties of both materials and on the interactions in the interface. We considered the cases of rigid flat to elastic rough and rigid rough to elastic rough contacts and two interface interaction laws: Coulomb friction and plastic flow. Depending on the conditions, we observe different regimes of wear from settling type to continuous and catastrophic wear. The dependencies of the wear volume on the normal force in the region of mild wear occur to be power-law dependencies. This behavior is congruent with experiments finding wear laws that deviate from Archard's law.

In the present work, we confined ourselves to the elastic behavior of the contacting materials (up to detaching of wear particle). This was mainly due to technical restrictions inherent to BEM. The concept proposed in the paper however, is independent of any underlying numerical procedure determining surface stresses and can equally be applied to other media, such as elastoplastic material.

\section{Acknowledgement}

This work has been conducted under partial financial support from the German Ministry for Research and Education BMBF (No. 13NKE011A).

Open Access: The articles published in this journal are distributed under the terms of the Creative Commons Attribution 4.0 International License (http:// creativecommons.org/licenses/by/4.0/), which permits unrestricted use, distribution, and reproduction in any medium, provided you give appropriate credit to the original author(s) and the source, provide a link to the Creative Commons license, and indicate if changes were made.

\section{References}

[1] Holmberg K, Erdemir A. Influence of tribology on global energy consumption, costs and emissions. Friction 5(3): 263-84 (2017)

[2] Wilkinson J M. Why do implants fail? In 15th EFORT Congress: A combined programme in partnership with the $B O A$, London, 4-6 June, 2014

[3] Wik A, Dave G. Occurrence and effects of tire wear particles in the environment-A critical review and an initial risk assessment. Environmental Pollution 157: 1-11 (2009)

[4] Cook L M. Chemical processes in glass polishing. Journal of Non-Crystalline Solids 120(1-3):152-71 (1990)

[5] Godet M. Third-bodies in tribology. Wear 136(1): 29-45 (1990)

[6] Reye Th. Zur Theorie der Zapfenreibung. Der Civilingenieur 4: 235-255 (1860)

[7] Kruschov M M. Resistance of metals to wear by abrasion, as related to hardness. London: Proc. Conf. Lubrication and wear, Institution Mech. Eng. 655-659 (1957)

[8] Krushchov M M, Babichev M A. Investigation of wear of metals. Moscow: Russian Academy of Sciences, 1960

[9] Archard J F. Contact and rubbing of flat surfaces. Journal of Applied Physics 24: 981-988 (1953)

[10] Archard J F, Hirst W. The wear of metals under unlubricated conditions. Proc. R. Soc. London A 236: 397-410 (1956)

[11] Rabinowicz E. Friction and Wear of Materials. Second Edition. John Wiley \& Sons, inc., 1995

[12] Kragelski I V. Friction and Wear. Butter Worth, 1965

[13] Akbarzadeh S, Khonsari M M. On the applicability of miner's rule to adhesive wear. Tribology Letters 63(2): 1-10 (2016)

[14] Meng H C, Ludema K C. Wear models and predictive equations: their form and content. Wear 181: 443-457 (1995)

[15] Aghababaei R, Warner D H, Molinari J-F. Critical length scale controls adhesive wear mechanisms. Nature Communications 7: 11816 (2016)

[16] Dimaki A, Shilko E, Psakhie S, Popov V L. Simulation of fracture using a mesh-dependent fracture criterion in the discrete element method. Facta Universitatis, Series: Mechanical Engineering 16(1): 41-50 (2018)

[17] Rabinowicz E. The effect of size on the looseness of wear fragments. Wear 2: 4-8 (1958)

[18] Dmitriev A I, Nikonov, A Y, Österle W. MD sliding simulations of amorphous tribofilms consisting of either $\mathrm{SiO}_{2}$ or carbon. Lubricants 4(3): 1-24 (2016) 
[19] Pohrt R, Li Q. Complete Boundary element formulation for normal and tangential contact problems. Physical Mesomechanics 17: 334-340 (2014)

[20] Aghababaei R, Warner D H, Molinari J-F. On the debrislevel origins of adhesive wear. Proceedings of the National Academy of Sciences 114(30): 7935-7940 (2017)

[21] Frérot L, Aghababaei R, Molinari J-F. A mechanistic understanding of the wear coefficient: From single to multiple asperities contact. Journal of the Mechanics and Physics of Solids 114: 172-84 (2018)

[22] Aghababaei R, Brink T, Molinari J-F. Asperity-level origins of transition from mild to severe wear, Physical Review Letters (2018), accepted paper, https://journals.aps.org/prl/ accepted/af070Y7eUba1b565e38540803364791de29e63f77.

[23] Li Q, Popov V L. On the possibility of frictional damping with reduced wear: A note on the applicability of Archard s law of adhesive wear under conditions of fretting. Physical Mesomechanics 20 (5): 91-95 (2017)

[24] Popov V L. Adhesive wear: Generalized Rabinowicz' criteria. Facta Universitatis, Series: Mechanical Engineering 16(1): 29-39 (2018)

[25] Popov V L. Contact Mechanics and Friction. Physical Principles and Applications. 2nd Edition. Berlin: Springer, 2017

[26] Bowden F P, Tabor D. The Friction and Lubrication of Solids. Clarendon Press, 2001

[27] Ciavarella M, Papangelo A. Discussion of "Measuring and understanding contact area at the nanoscale: A review"

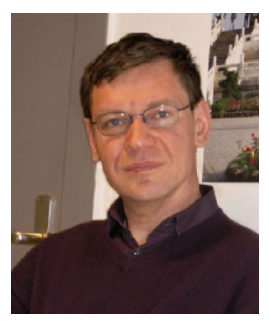

Valentin L. POPOV. He is full professor at the Berlin University of Technology. He studied physics and obtained his doctorate in 1985 from the Moscow State Lomonosov University. 1985-1998 he worked at the Institute of Strength Physics and Materials Science of the Russian Academy of Sciences and was a guest professor in the field of theoretical physics at the University of Paderborn (Germany) from 1999 to 2002. Since 2002 he is the head of the Department of System Dynamics and the Physics of Friction at the Berlin University of Technology. He has published over 300 papers in leading international journals and is the author of the book "Contact Mechanics and Friction: Physical Principles and Applications" which appeared in three editions in
(Jacobs T D B, Ashlie Martini A, ASME Appl. Mech. Rev. 69(6): 060802 (2017)). Applied Mechanics Reviews. ASME International. 69(6): 065502 (2017)

[28] Pohrt R, Popov V L. Contact mechanics of rough spheres: Crossover from fractal to Hertzian behavior. Advances in Tribology 2013:1-4 (2013)

[29] Popov V L, Pohrt R, Li Q. Strength of adhesive contacts: Influence of contact geometry and material gradients. Friction 5(3):308-25 (2017)

[30] Popov V L. Generalized Rabinowicz' criterion for adhesive wear for elliptic micro contacts, AIP Conference Proceedings 1909: 020178 (2017)

[31] Popov V L, Smolin I Yu, Gervé A, Kehrwald B. Simulation of wear in combustion engines. Computational Materials Science 19: 285-291 (2000)

[32] Popov V L, Psakhie S G. Numerical simulation methods in tribology. Tribology International 40(6): 916-23 (2007)

[33] Scherge M, Shakhvorostov D, Pöhlmann K. Fundamental wear mechanism of metals. Wear 255: 395-400 (2003)

[34] Popov V L. Analytic solution for the limiting shape of profiles due to fretting wear. Scientific Reports 4: 3749 (2014).

[35] Dmitriev A I, Voll L B, Psakhie S G, Popov V L. Universal limiting shape of worn profile under multiple-mode fretting conditions: theory and experimental evidence. Scientific Reports 6: 23231 (2016)

[36] Rhee S K. Wear equation for polymers sliding against metal surfaces. Wear 16(6):431-45 (1970)

[37] Rabinowicz E. The least wear. Wear 100: 533-541 (1984).

German, English, Chinese, and Russian. He is the member of editorial boards of many international journals and is organizer of more than 20 international conferences and workshops over diverse tribological themes. Prof. Popov is honorary professor of the Tomsk Polytechnic University, of the East China University of Science and Technology, and of the Changchun University of Science and Technology and Distinguished Guest Professor of the Tsinghua University. His areas of interest include tribology, nanotribology, tribology at low temperatures, biotribology, the influence of friction through ultrasound, numerical simulation of contact and friction, research regarding earthquakes, as well as topics related to materials science such as the mechanics of elastoplastic media with microstructures, strength of metals and alloys, and shape memory alloys. 


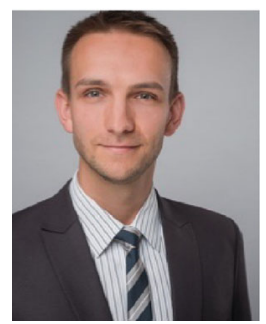

Roman POHRT. He is researcher at the Berlin University of Technology. He studied physical engineering science with special focus on simulation and optimization of discrete and continuous problems. Since he joined the group of Prof. V. Popov in 2010, he has been conducting experimental and numerical research on a variety of tribology related industry problems. In his $\mathrm{PhD}$ thesis R. Pohrt focussed on linking scales in the elastic contact of fractal rough surfaces, for which he was awarded by the German Tribological Society in 2013. R. Pohrt has authored a series of influential papers on different tribological problems, applying and extending stateof-the-art numerical methods. His areas of interest include contact mechanics, rail-wheel-interaction of trains, manufacturing technology, lubrication and more generally the influence of surface topography on tribological phenomena. 